\title{
A study of visually directed throwing in the presence of multiple distance cues
}

\author{
DAVID W. EBY and JACK M. LOOMIS \\ University of California, Santa Barbara, California
}

\begin{abstract}
Visually directed throwing in a multicue situation was investigated using two related motoric activities, underhand and overhand throwing. Subjects threw balls at targets ranging from 5 to $25 \mathrm{~m}$ away in a large open field. In most conditions, the subjects viewed the target and then, with vision occluded, waited for some specified delay before throwing. There was no systematic effect of delay on either accuracy or variability of the throws, and mean throwing error was encountered mainly in the form of underthrowing at the far distances. The data were fit with both power and hyperbolic functions. This analysis indicated that thrown distance is a negatively accelerated function of target distance over the range studied. The parameters characterizing the negative accelerations were highly correlated for the two types of throwing over the 8 subjects.
\end{abstract}

Thomson (1983) reported a series of experiments on the visual control of locomotion. The results of these experiments not only reveal much about the internal processes involved in the control of motor activity, but also have implications for the study of visual space perception. In several of his experiments, Thomson had subjects view a target placed on the ground at an egocentric distance ranging from 3 to $21 \mathrm{~m}$. When ready, the subject closed his eyes and attempted to walk the distance to the target. Thomson found that the average walked distance was in virtually perfect agreement with the target distance over the full range of distances studied; the mean signed error of response was less than $20 \mathrm{~cm}$ for each of the distances tested. The analysis of the response variability provided equally surprising results. If subjects were able to complete the response within $8 \mathrm{sec}$ (corresponding to a distance of about $12 \mathrm{~m}$ ), the variability of the response did not vary with target distance. However, the variability of the responses increased precipitously for distances greater than about $12 \mathrm{~m}$, which required more than $8 \mathrm{sec}$ at the average pace of the subjects.

Thomson was able to show that this drop in precision was related not so much to the target distance as to the time that had elapsed between the start of visual occlusion and completion of the response. In one experiment, the subject remained at the start point for a specified delay with eyes closed before walking toward the target. Delays of up to $10 \mathrm{sec}$ had no effect on performance for distances less than $6 \mathrm{~m}$, but performance suffered a drastic decline in precision for distances greater than $6 \mathrm{~m}$, when the response took longer than $8 \mathrm{sec}$ to complete. Converging evidence for the conclusion that elapsed time without vision determined the precision of the responses came

The authors are grateful to John M. Foley, Jose A. Da Silva, and two reviewers for comments on drafts of this paper and to Stephen Wright for his assistance in collecting data. Requests for reprints should be sent to either author at the Department of Psychology, University of California, Santa Barbara, CA 93106. from an experiment in which the subject ran, rather than walked, to the target. After excluding trials in which the response took longer than $8 \mathrm{sec}$, Thomson found that the variability of the responses increased only slightly with distance.

Thomson's (1983) interpretation of these results was that visually guided locomotion is controlled by either of two internal processes. In the case of relatively simple locomotor activity, such as blind walking to a target within a distance of $6 \mathrm{~m}$, the subject is able to assemble during preview a motor program that endures without loss of integrity for a period considerably longer than $8 \mathrm{sec}$. For more complex motor acts, such as blind walking to much more distant targets, motor programs prepared in advance are not effective, and the subject needs to use some form of visually acquired internal representation of surrounding space to generate the motoric responses concurrently with their execution; this type of representation remains high in fidelity for about $8 \mathrm{sec}$ and then rapidly loses articulation.

The primary purpose of the present experiment was to test the generality of Thomson's (1983) findings. The task involved throwing a baseball to targets at different egocentric distances with vision occluded. The procedure was quite similar to that used by Thomson; after viewing the target to establish its distance, the subject underwent a predetermined period of visual occlusion and then threw the ball at the target. The egocentric distances ranged from 5 to $25 \mathrm{~m}$, and the delays of visual occlusion ranged from 0 to $54 \mathrm{sec}$. Two types of response-underhand and overhand throwing-were investigated.

\section{METHOD}

\section{Stimuli and Materials}

The experiment took place in an unfamiliar section of a flat grassy field of large dimensions $(250 \times 450 \mathrm{~m})$. A measuring rope, marked at $10-\mathrm{cm}$ intervals, was anchored at the throwing line. Seven yellow plywood squares with widths of $7.5,10.012 .5,15.0,17.5$, 20.0 , and $22.5 \mathrm{~cm}$ were used as targets; they were mounted on ply- 
wood bases. Foam packing material was attached to the squares to deaden any sound that would result from a direct hit. A blinder device, designed to occlude peripheral and frontal vision, was attached to adjustable eyeglass frames and could be easily flipped up and down by the subject. The balls used in throwing were baseballs of standard size (diameter $=7.25 \mathrm{~cm}$ ) and weight $(142 \mathrm{~g}$ ).

\section{Subjects}

Eight male students volunteered to participate as paid subjects (mean age $=22$ ). As judged by self-report, all were experienced and proficient at throwing a ball both overhand and underhand. All but 1 of the subjects either were currently participating or had participated in intercollegiate athletics. The other was an active participant in intramural athletics. All but 1 of the subjects had nor$\mathrm{mal}$ or corrected-to-normal visual acuity $(20 / 20)$ as measured by a Keystone Orthoscope. The remaining subject had normal acuity in his right eye and 20/200 in his left eye.

\section{Procedure}

The experiment proceeded in three phases-warm-up, testing, and conclusion. During the warm-up phase, which was conducted in an area of the field removed from that used during testing, the subject played catch with the experimenter, using both underhand and overhand throwing styles. The subject was then fitted with the blinder device. He then continued throwing with the blinder in both the up and down positions until he was comfortable with the entire task. The warm-up phase lasted an average of $15 \mathrm{~min}$.

The testing phase of the experiment consisted of 32 trials, 20 for overhand throws (five distances at each of four delays) and 12 for underhand throws (three distances at each of four delays). The egocentric target distances were $5,10,15,20$, and $25 \mathrm{~m}$ for overhand throws and 5,10 , and $15 \mathrm{~m}$ for underhand throws. The delay values were 0,6,18, and 54 sec. Each subject received a different random order of the experimental conditions.

During this phase, the experimenter's assistant placed the stimulus target prior to each throw and measured the distance of the throw, both while the subject's eyes were closed. While the subject viewed the target, the assistant remained $25^{\circ}$ to one side of the line defining the target distances.

At the beginning of each trial, the assistant placed one of the targets at the appropriate distance. The size of the target was determined by random assignment to minimize the subject's use of familiar size as a potential cue to distance. After being informed of the type of throw to be used, the subject attended to the single target placed within his field of view. When ready, he flipped the occluder to the down position, using his free hand, and the experimenter began timing the interval of visual occlusion. At the end of the interval the experimenter said "Throw" and the subject attempted to throw to the target. (In the case of zero delay, the subject simply flipped the occluder down and threw.) After the subject's first throw, exactly the same procedure was followed for the second throw, using the same combination of distance, delay, and type of throw. Immediately after the second throw, the subject reported which attempt he judged, on the basis of motoric and kinesthetic information, to be more accurate. At no time during testing did the subject receive visual or verbal feedback about the accuracy of a throw. The testing phase lasted approximately $75 \mathrm{~min}$.

The subject concluded the session by removing the blinders and, with unrestricted vision, attempting to throw beyond the maximum distances for overhand (25 m) and underhand (15 m) throwing. All subjects easily threw farther than these distances.

\section{RESULTS}

On each trial the subject made two throws in succession and then judged which he thought to be more accurate. Analysis revealed no difference in accuracy between the two throws, which were then averaged to give

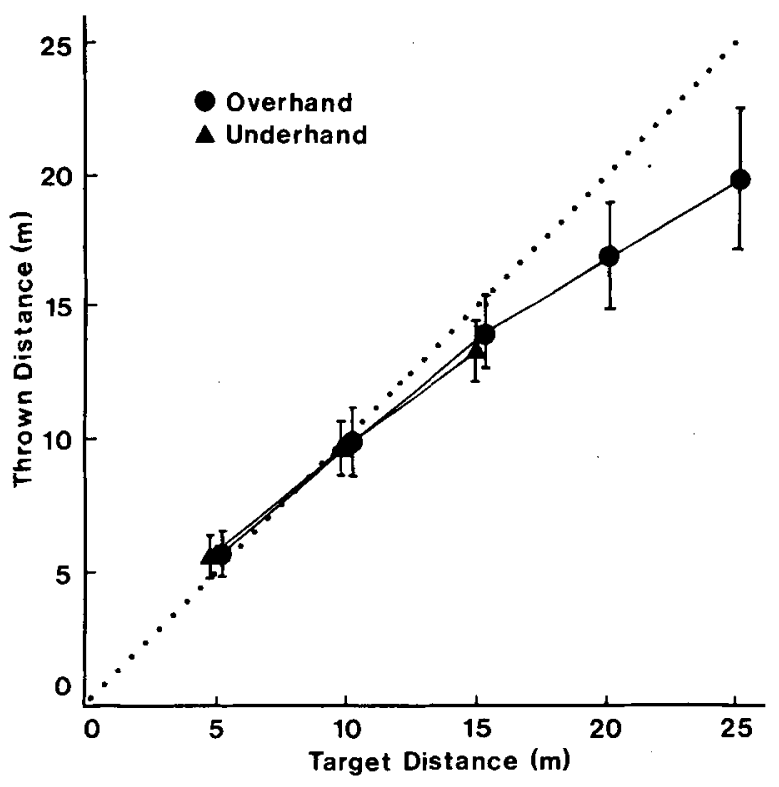

Figure 1. Mean thrown distance (averaged across delay, subject, and repetition) as a function of physical target distance and type of throw. The error bars represent $\pm 1 S D$.

a mean throw for each subject in each condition. Figure 1 plots the mean thrown distance and standard deviation (averaged across subjects and delays) as a function of target distance and type of throw. Table 1 gives the mean and standard deviation of the signed error for each condition, averaged over the 8 subjects.

The effects of delay and distance on the signed error were assessed by separate analyses of variance for underhand and overhand throws. Because the response variances for the larger target distances were greater than those for the shorter ones, a square root transformation was applied to both stimulus (target) and response (throw) distances prior to the computation of signed error; this resulted in roughly homogeneous variance across conditions. Not surprisingly, the tests for a main effect of distance were highly significant for both overhand and underhand throwing $[F(4,28)=2.44, p<.001$, and $F(2,14)$

Table 1

Signed Error of Thrown Distances (in meters): Mean and Standard Deviation for Two Types of Throw, Five Distances, and Four Delays, Averaged Across Subjects

\begin{tabular}{|c|c|c|c|c|c|c|c|c|}
\hline \multirow[b]{3}{*}{ Distance } & \multicolumn{8}{|c|}{ Delay } \\
\hline & \multicolumn{2}{|c|}{$0 \mathrm{sec}$} & \multicolumn{2}{|c|}{$6 \sec$} & \multicolumn{2}{|c|}{$18 \mathrm{sec}$} & \multicolumn{2}{|c|}{$54 \mathrm{sec}$} \\
\hline & Mean & $S D$ & Mean & $S D$ & Mean & $S D$ & Mean & $S D$ \\
\hline \multicolumn{9}{|c|}{ Overhand Throw } \\
\hline $\begin{array}{r}5 \\
10 \\
15 \\
20 \\
25\end{array}$ & $\begin{array}{r}0.29 \\
-0.08 \\
-0.28 \\
-2.47 \\
-4.50\end{array}$ & $\begin{array}{l}0.81 \\
1.34 \\
1.22 \\
1.95 \\
2.83\end{array}$ & $\begin{array}{r}0.36 \\
0.03 \\
-1.44 \\
-3.06 \\
-6.24\end{array}$ & $\begin{array}{l}0.98 \\
1.18 \\
1.39 \\
1.52 \\
2.14\end{array}$ & $\begin{array}{r}0.82 \\
-0.25 \\
-1.64 \\
-4.46 \\
-4.97\end{array}$ & $\begin{array}{l}0.85 \\
1.45 \\
1.56 \\
1.71 \\
3.11\end{array}$ & $\begin{array}{r}1.11 \\
-0.33 \\
-0.84 \\
-2.36 \\
-5.32\end{array}$ & $\begin{array}{l}0.98 \\
1.43 \\
1.43 \\
2.96 \\
2.63\end{array}$ \\
\hline \multicolumn{9}{|c|}{ Underhand Throw } \\
\hline $\begin{array}{r}5 \\
10 \\
15\end{array}$ & $\begin{array}{r}0.35 \\
-0.08 \\
-1.07\end{array}$ & $\begin{array}{l}0.67 \\
0.94 \\
1.74\end{array}$ & $\begin{array}{r}0.77 \\
-0.37 \\
-1.68\end{array}$ & $\begin{array}{l}0.78 \\
0.93 \\
0.96\end{array}$ & $\begin{array}{r}0.52 \\
-0.94 \\
-2.04\end{array}$ & $\begin{array}{l}1.12 \\
0.77 \\
1.24\end{array}$ & $\begin{array}{r}0.81 \\
0.05 \\
-2.02\end{array}$ & $\begin{array}{l}0.57 \\
1.87 \\
1.23\end{array}$ \\
\hline
\end{tabular}


$=18.24, p<.001$, respectively]. There were no main effects of delay [overhand, $F(3,21)=2.72, p>.05$; underhand, $F(3,21)=1.12, p>.05]$. There was a distance $X$ delay interaction for overhand throwing $[F(12,84)=$ $2.18, p<.05]$, but not for underhand throwing $[F(6,42)$ $=.92, p>.05]$. These analyses indicate an effect of distance on signed error, but no effect of delay. With the design we used, we were unable to test the effects of these variables on the variability of the thrown distances; however, a glance at Table 1 clearly indicates a large effect of distance and no systematic effect of delay. In addition, we computed the standard deviations of the two responses given by each subject in each condition and observed no tendency for response variability to increase with delay. Thus, whether one looks at within-subjects or between-subjects variability, there is no systematic effect of delay, a result that contrasts markedly with Thomson's (1983) finding that variability of the responses increased precipitously at delays of about $8 \mathrm{sec}$. Incidentally, the average between-subjects standard deviation was 2.82 times the average within-subjects standard deviation, averaging over the 32 different conditions.

In analyzing the functional relationship between physical and thrown distance, we used two equations that have been used to express how apparent distance varies with physical distance: the power function (Baird, 1970; Künnapas, 1960) and the hyperbolic function (Foley, 1977, 1980, 1985; Gilinsky, 1951; also see the Appendix). The psychophysical power function is of the form

$$
D^{\prime}=k D^{n} \text {, }
$$

where $D$ is physical distance, $D^{\prime}$ is apparent distance, and $k$ is a scaling factor. The exponent $n$ is the more important parameter specifying the relationship between apparent distance and physical distance. One limitation of the power function as a way of relating apparent distance to physical distance is that it does not correctly represent the apparent distance of very distant targets. Using several converging methods, Gilinsky (1951) found that apparent distance is asymptotic for extremely large physical distances. Because the power function is nonasymptotic, one might question its appropriateness for representing apparent distance.

A virtue of the hyperbolic function is that it does approach an asymptotic value. Expressed in linear (inverse) form, the hyperbolic function is

$$
1 / D^{\prime}=E+F / D,
$$

where $E$ and $F$ are constants specifying the intercept and slope (Foley, 1977, 1980, 1985). In the special case where physical and apparent distance are equal, $E$ has a value of zero and $F$ has a value of one.

The two equations were fitted to the data of each subject, averaged over delay; in this case $D$ represented physical distance to the target and $D^{\prime}$ represented thrown, rather than apparent, distance. Both functions were fitted by first transforming the stimulus and response values to yield a linear relation and then finding a line of best fit by the least squares method. For the power function, target distance and thrown distance were transformed logarithmically; for the hyperbolic analysis, the reciprocals of thrown and target distance were taken (Foley, 1985). Table 2 gives the parameters and goodness-of-fit (root-mean-square [RMS]) values of the two functions by subject and by type of throw. The correlations between the values of $n$ and $F$ across subjects were $r=.89$ for overhand throwing and $r=.96$ for underhand throwing. The high correlation between individual $n$ and $F$ values for both types of throws and the similarity of the mean RMS values indicate that both functions fit the data equally well over the range of distances studied here.

The parameter values also reveal an interesting fact about individual differences in performance. Over the 8 subjects, the power function exponents for overhand throwing exhibit a correlation of $r=.80$ with those of underhand throwing: the same comparison for the inverse hyperbola slopes yields a correlation of $r=.60$. Excluding Subject $H$, these values increased to .93 and .90 , respectively. Since both the exponent and the slope are measures of negative acceleration, this result indicates that the negative accelerations of the response functions across subjects are similar for overhand and underhand throwing.

\section{DISCUSSION}

Despite the attention it has received, the problem of egocentric distance perception still eludes our understanding, as indicated by the lack of agreement among different methods for scaling apparent distance in multicue environments. Direct scaling methods have resulted in considerable variation in the exponents of the best-fitting power functions, both between individuals within an ex-

Table 2

Parameters and Root-Mean-Square (RMS)

\begin{tabular}{|c|c|c|c|c|c|c|}
\hline \multirow[b]{2}{*}{ Subject } & \multicolumn{3}{|c|}{ Power } & \multicolumn{3}{|c|}{ Inverse Hyperbola } \\
\hline & $k$ & $n$ & RMS & $E$ & $F$ & RMS \\
\hline \multicolumn{7}{|c|}{ Overhand Throw } \\
\hline A & 1.41 & .86 & .277 & .012 & .83 & .278 \\
\hline B & 1.41 & .86 & .669 & .013 & .81 & .663 \\
\hline $\mathrm{C}$ & 1.58 & .73 & .114 & .029 & .85 & .276 \\
\hline D & 2.45 & .63 & .866 & .029 & .64 & .710 \\
\hline $\mathbf{E}$ & .99 & .96 & .456 & .004 & 1.07 & .415 \\
\hline $\mathbf{F}$ & 2.79 & .59 & .306 & .036 & .52 & .630 \\
\hline G & 1.58 & .82 & .250 & .015 & .79 & .407 \\
\hline $\mathbf{H}$ & 1.23 & .84 & .744 & .017 & .98 & .580 \\
\hline Mean & 1.68 & .79 & .460 & .019 & .81 & .495 \\
\hline \multicolumn{7}{|c|}{ Underhand Throw } \\
\hline A & 1.51 & .84 & .028 & .018 & .78 & .160 \\
\hline B & 1.25 & .89 & .167 & .014 & .87 & .296 \\
\hline C & 1.23 & .85 & .062 & .021 & .93 & .176 \\
\hline D & 2.45 & .61 & .304 & .041 & .57 & .154 \\
\hline $\mathbf{E}$ & 1.02 & .95 & .183 & .008 & 1.01 & .275 \\
\hline F & 2.25 & .63 & .485 & .039 & .62 & .364 \\
\hline G & 1.39 & .85 & .229 & .016 & .85 & .165 \\
\hline $\mathbf{H}$ & 2.01 & .68 & .544 & .041 & .62 & .802 \\
\hline Mean & 1.64 & .79 & .250 & .025 & .78 & .303 \\
\hline
\end{tabular}
Values for Two Fitted Functions 
periment and between similar experiments (Da Silva, 1985; Da Silva \& Fukusima, 1986); the exponents characterizing scales for outdoor environments have tended to be somewhat less than unity (Baird, 1970; Da Silva, 1985; Da Silva \& Fukusima, 1986; Teghtsoonian \& Teghtsoonian, 1970). In contrast, the method of equal-appearing depth intervals used by Gilinsky (1951) resulted in a scale of apparent distance with much greater negative acceleration. Against this background of research indicating that apparent distance in natural outdoor environments tends to be a negatively accelerated function of distance, with considerable variation from subject to subject, the results obtained by Thomson (1983) are indeed surprising. After a period of visual preview, his subjects were able to walk blindly to targets up to $20 \mathrm{~m}$ away virtually without constant error, and thus without any evidence of negative acceleration in their responses as a function of distance. Although it is tempting to conclude from Thomson's work that egocentric distance perception is accurate for distances of up to $20 \mathrm{~m}$, to do so would be to ignore the seemingly contradictory evidence provided by the studies mentioned above. A proper theory of perceived egocentric distance or of visual space perception in general would provide an understanding of all of the responses used by subjects to indicate their knowledge of space. Conversely, in view of the difficulties encountered so far in research on visual space perception, what may be needed is a systematic study of many different responses, in the hope that invariances in the data obtained will point the way to a comprehensive theory of visual space perception and performance (Foley, 1980, 1985).

In the present experiment, we studied underhand and overhand throwing to targets ranging from 5 to $25 \mathrm{~m}$ away from the subject. In contrast to Thomson's (1983) finding of accurate performance with walking, our results, averaged over the delay variable, indicated underthrowing for distances greater than $10 \mathrm{~m}$, with substantial constant errors at 15 and $25 \mathrm{~m}$. When the data of each subject were fitted by power and hyperbolic functions, 7 of the 8 subjects had functions with negative acceleration (downward curvature). The surprising result here was that the parameters characterizing acceleration were highly correlated between the two types of throwing, even though different ranges of distance were used and the two types of throwing involve quite different muscle groups. Before any inference can be made about the accuracy of egocentric distance perception under these conditions, further work is needed to establish the connection between throwing and perceived egocentric distance. However, the data speak for themselves in indicating that not all highly practiced motoric responses result in the degree of accuracy obtained by Thomson.

Our second concern was to assess the effect of delaying the subject's throwing response after the onset of visual occlusion. Thomson (1983) found that if the target distance was greater than $5 \mathrm{~m}$ and if completion of the subject's response took longer than $8 \mathrm{sec}$ following the onset of visual occlusion, the variability of the response increased dramatically. On the basis of the results from a series of experiments, Thomson hypothesized that, during viewing, the subject forms a visual representation of surrounding space, a representation that then remains high in fidelity for about $8 \mathrm{sec}$ following the onset of visual occlusion. It is this representation that guides the subject's formulation and execution of very complex motoric responses, such as blind walking to distant targets. However, Thomson also hypothesized that for targets within $6 \mathrm{~m}$, the subject is able to prepare a motor program for blind walking to the target and that this type of representation is more durable than the other type, although less flexible. In the present experiment, the subject made a throw to targets ranging from 5 to $25 \mathrm{~m}$ away with delays of $0,6,18$, and $54 \mathrm{sec}$ following the onset of visual occlusion. In contrast to Thomson's finding for distances greater than $6 \mathrm{~m}$, delaying the response here had neither sizable nor systematic effects on the signed error or its variability: subjects performed with about the same accuracy and precision whether the delay was $54 \mathrm{sec}$ or $0 \mathrm{sec}$. However, it is not difficult to see a critical difference between the two experiments. Even though subjects were responding to distant targets in the present experiment, the complexity of the response was minimal in comparison with that of blind walking to a distant target. Thus, subjects in the present experiment may have formulated motor programs for throwing during the period of visual preview, as Thomson hypothesized for blind walking to nearby targets. Under the premise that motor programs do underlie the throwing performance studied here, the data obtained imply that such motor programs are indeed quite durable.

\section{REFERENCES}

BAIRD, J. C. (1970). Psychophysical analysis of visual space. Oxford: Pergamon Press.

DA SilvA, J. A. (1985). Scales for perceived egocentric distance in a large open field: Comparison of three psychophysical methods. American Joumal of Psychology, 98, 119-144.

DA Silva, J. A., Fuxusima, S. S. (1986). Stability of individual psychophysical functions for perceived distance in natural indoor and outdoor settings. Perceptual \& Motor Skills, 63, 891-902.

FoLeY, J. M. (1977). Effect of distance information and range on two indices of visually perceived distance. Perception, 6, 449-460.

Foley, J. M. (1980). Binocular distance perception. Psychological Review, 87, 411-433.

FoLEY, J. M. (1985). Binocular distance perception: Egocentric distance tasks. Joumal of Experimental Psychology: Human Perception \& Performance, 11, 133-149.

Giunssky, A. S. (1951). Perceived size and distance in visual space. Psychological Review, 58, 460-482.

HooD, D. C. (1978). Psychophysical and physiological tests of proposed physiological mechanisms of light adaptation. In J. Arming ton, J. Krauskopf, \& B. Wooten (Eds.), Visual psychophysics: Its physiological basis. New York: Academic Press.

KüNNAPAS, T. (1960). Scales for subjective distance. Scondinavian Journal of Psychology, 1, 187-192.

NAKA, K. I., \& Rushton, W. A. H. (1966). S-potentials from lumi- 
nosity units in the retina of fish (cyprinidae). Journal of Physiology, 185, 587-599.

Teghtsoonian, R., Teghtsoonian, M. (1970). Scaling apparent distance in a natural outdoor setting. Psychonomic Science, 21, 215-216.

Thomson, J. A. (1983). Is continuous visual monitoring necessary in visually guided locomotion? Journal of Experimental Psychology: Human Perception \& Performance, 9, 427-443.

\begin{abstract}
APPENDIX
The hyperbolic function expressing apparent distance $\left(D^{\prime}\right)$ as a function of physical distance has appeared in at least four different forms in research on vision. Foley $(1977,1980,1985)$ has used two forms: $D^{\prime}=D /(E D+F)$ and its inverse form $1 / D^{\prime}$
\end{abstract}

$=E+F / D$. The meanings of $E$ and $F$ as $y$-intercept and slope are given when $D$ and $D^{\prime}$ are plotted as reciprocals. Gilinsky used $D^{\prime}=A D /(A+D)$, where $A$ is the asymptotic value of $D^{\prime}$ for $D$ equal to infinity. The fourth form is borrowed from a similar expression for response versus intensity functions of receptors and higher order retinal neurons (Hood, 1978; Naka \& Rushton, 1966) and is given by $D^{\prime}=A D /(D+D \sigma)$, where again $A$ is the asymptotic value of $D^{\prime}$. The so-called semisaturation constant, $D \sigma$, is the value of $D$ at which $D^{\prime}$ is half the value of $A$; changes in $D \sigma$ induce a rescaling of the $D$ dimension. Gilinsky's equation is a special one-parameter case of Foley's equation where $A=1 / E$ and $F=1$. The fourth expression is equivalent to Foley's with $A=1 / E$ and $D \sigma$ equal to $F / E$.

(Manuscript received December 9, 1985;

revision accepted for publication November 10, 1986.) 